in tissue culture), it is in the context of the whole organism; he then reviewed the eleven real facts about plant ageing discovered in the past 85 years. Professor T. Villiers (University of Natal) outlined his intriguing new theory of seed ageing, which states that seeds survive in the soil for long periods not because they are completely dormant, but because they are metabolically active and can carry out essential repair and maintenance.

Echoing von Hahn's scepticism, Dr P. Wilson (Imperial Cancer Research Fund, London) suggested that previous work on quantitative changes in enzymatic activity in ageing tissues had been confusing and unprofitable. Her studies on thirteen enzymes in kidney, lung and liver from C57 mice in four age groups had shown only that (at least in mice) sex modifies the effects of ageing, and that some changes probably occur in mitochondrial respiratory enzymes. Dr Wilson and her colleague Dr C. Rowlatt agreed that ageing in tissues is initially a focal process and, like many other participants, felt that various cells in various organs probably aged in different ways, even in different individuals of the same species.

Dr L. Robert (Centre National de la Recherche Scientifique, Paris) considered that ageing in extracellular materials is caused by two separate phenomena-a continuous shift in the proportions of the various extracellular molecules synthesized, and the modification of the extracellular matrix with age, with feedback affecting the metabolism of the cells themselves. Dr U. Schmidt (University Charity Clinic, Berlin) also felt that ageing is not merely a process of fibrosis or sclerosis, but the result of variations in rates of synthesis and degradation. Dr P. Leuenberger (University Eye Clinic, Geneva) described an age-correlated thickening of the capillary basement membrane in the retinas of spiny mice and rats, and suggested that similar changes in pathological conditions, such as diabetes, might be caused by the acceleration of a normal process rather than by the induction of new phenomena. Dr W. de Priester (University of Leiden) described the accumulation of dense lysosomal bodies in the midgut epithelium of the fiy, Calliphora, and pointed out that insects are of great value in ageing studies because there is virtually no cell renewal in the adult.

$\mathrm{Mr}$ A. Payne (University of Edinburgh) discussed the progressive decline with age in the ability of mice to produce specific antibody. Because young and old cells are equivalent in their ability to mount a response in young irradiated hosts, and because young cells cannot significantly improve the response of old irradiated recipients, he considered that this is an organismal phenomenon caused by some cellular, tissue structural or hormonal deficiency. Professor D. Bellamy (University of Cardiff) discussed the thymus as a model for studies on ageing and concluded that cortisol-induced involution of the thymus might represent the acceleration of a normal process-by accelerating cell death without inducing a corresponding increase in cell proliferation.

The overall impression remaining from this conference is, first, that there is no general agreement on what ageing really is and, second, that it is difficult to make comparisons between aged and non-aged cells, tissues or organisms. Are the aged a physiological elite, survivors of the functional failures or parasitic infections which normally kill at an earlier stage? Does ageing occur in the absence of disease? Dr Schmidt surprised the audience with his report that all 3,000 "healthy" over-65s examined in the ageing study had two to five diseases, many of which were treated successfully (so some good came out of the work). Dr Robert made the one irrefutable point-that at each meeting when he saw his colleagues in the ageing field again, he felt that they seemed a little older than at the previous conference.

\section{Regulation of the Tryptophan Operon}

THE set of five contiguous genes which specify enzymes involved in the biosynthesis of tryptophan in the Escherichia coli chromosome constitute an operon for the expression of these genes (designated Operator, $\operatorname{tryp} E, D, C, B, A)$ is coordinately controlled by the intracellular concentration of tryptophan. When this amino-acid is present the genes are switched off; conversely when the cells are starved for tryptophan this set of genes is expressed. Moreover, as several groups have shown, tryptophan seems to exert its regulatory effect by first interacting with the product of a regulatory gene trypR which is not adjacent to the operon.

Because the transcription of the tryp operon starts near the beginning of the $E$ gene and for other genetic reasons it has often been suggested that the polypeptide chain specified by this first structural gene of the operon also plays a part in the feedback control of the entire operon. The latest experiments of Hiraga and Yanofsky, which are reported in next Wednesday's Nature New Biology (May 10) indicate, however, that neither intact $E$ gene messenger RNA nor intact $E$ gene polypeptide are essential for the regulated expression of the other four genes $(\operatorname{tryp} D, C, B, A)$ of the operon.

Hiraga and Yanofsky have reached
SOLAR MAGNETISM Sunspot Variations

by our Cosmology Correspondent

VARIATIONS in the magnetic field of the Sun may be explained in terms of the dispersion of sunspot groups over the solar surface. J. Tuominen, of the University of Helsinki, has now presented evidence, in the form of sunspot counts, which supports his theory that regular changes in the magnetic field of the Sun occur because the following part of a bipolar sunspot group always disperses more rapidly than the leading part (Astrophys. Lett., 10, 175; 1972).

The observations which led to this suggestion were made by Severny (Nature, 224, 53; 1970), who found that the intensity curve of the solar magnetic field is similar to that of magnetic stars, and in particular that the Sun behaves like a quadrupole magnetic rotator. Tuominen pointed out that because of the rapid dispersion of the following part of sunspot groups there must be a widely distributed general magnetic flux balancing the strong localized field in the leading parts, and that variations associated with the sunspot cycle could explain Severny's observations (Nature, 228, 1179; 1972).

this somewhat surprising conclusion from analysis of a deletion mutant strain designated $\triangle$ trypE5. In this strain, in spite of the absence of a complete trypE gene, the expression of the genes $\operatorname{tryp} D, C, B, A$ is repressed when tryptophan is added and derepressed when tryptophan is removed from the culture medium. In other words, in spite of lacking at least part of the $E$ gene the remainder of the operon in cells of this strain is sensitive to feedback regulation.

But just how much of the trypE gene is missing? By comparing the time taken to transcribe the tryp operon in a strain in which the operon is intact with the time taken in the strain carrying the $\triangle$ trypE5 deletion, Hiraga and Yanofsky have shown that it takes about 50 seconds longer to transcribe the intact operon than to transcribe the deleted operon. If transcriptase polymerizes RNA at a rate of about thirtythree bases a second some 1,650 bases of the trypE gene must be missing and the entire gene comprises only about 1,700 base pairs. In short, 97 per cent of the trypE gene seems to be deleted in $\triangle$ trypE5, but the other genes of the operon are still subject to feedback control. It is difficult therefore to avoid the conclusion that this regulation does not depend upon the production of an intact $E$ gene messenger. 